\title{
Know thyself
}

\author{
MARIANO A. GARCIA-BLANCO ${ }^{1,2}$ \\ ${ }^{1}$ Department of Biochemistry and Molecular Biology, University of Texas Medical Branch, Galveston, Texas 77555-0144, USA \\ ${ }^{2}$ Program in Emerging Infectious Diseases, Duke-NUS Graduate Medical School, Singapore 169857
}

The realization that RNA molecules could be enzymes as well as genetic material suggested that RNAs were likely the earliest genomes to evolve on earth. The implications for evolution are many and very interesting, but here I will focus on one that I consider the most interesting: the central role of RNA in the ability of genomes (even latecomer DNA genomes) to encode systems that recognize signatures of foreign genomes and by inference to know themselves.

My personal interest in this area goes back to my $\mathrm{PhD}$ studies on the interferon system and in particular the finding that interferons could selectively inhibit the expression of SV40 genes from an incoming virus (or plasmid) but not from integrated copies of the same. In fact, the interferon system could discriminate between endogenous and exogenous genes, even when driven by cellular promoters, (published and unpublished results with Peter Lengyel at Yale University). After excluding many trivial explanations for these phenomena we concluded that the interferon system could determine the provenance of the genetic material and would suppress the expression of exogenous genes. Although at the time we did not unravel the precise mechanism of discrimination, these observations led me to an interest in this type of self vs. non-self recognition, and, given the central role of double stranded RNA (dsRNA) in the interferon system, to suspect that RNA played a critical role in the evolution of this recognition. Here I will present my views on how this aspect of RNA biology has been and continues to be an exciting area of investigation.

The role of RNA in self vs. non-self discrimination is evident in many ancient immune mechanisms. In several kingdoms of life the RNA interference system, which I use here generically, uses several types of very small RNAs to guide the restriction of gene expression from both RNA and DNA foreign genomes, whether these are invading genomes (e.g., pathogenic viruses) or passengers in the host genome (e.g., transposons). Indeed, there is strong evidence that this system can also recognize endogenous sequences as non-threatening, and license their expression (e.g., work of Mello et al). Taken together the work of many has shown

\footnotetext{
Corresponding author: maragarc@utmb.edu

Article and publication date are at http://www.rnajournal.org/cgi/doi/10. 1261/rna.050138.115. Freely available online through the RNA Open Access option.
}

that this fascinating RNA-based recognition system can provide both innate and acquired types of immunity.

In the last decade or so the discovery of the Clustered regularly interspaced short palindromic repeats (CRISPR) and Cas nucleases, the CRISPR-Cas system, in bacteria and archaea revealed another RNA-centric acquired immune system. Just like in RNA interference, the CRISPR-Cas system uses RNAs as guides that read and recognize foreign sequences and can lead to cleavage and inactivation of the offending genome. As difficult as it is to avert one's attention from the bewildering applications of this system, the emphasis here is on the remarkable biology that uses RNA to protect self vs. non-self.

Although less is known about how RNA editing and modification systems work in this regard, there is evidence that supports a role in immunity. Viral sequences are edited differently from cellular RNAs, which can lead to increased recognition of these as foreign by sensors and in vertebrates there is a direct connection to inflammatory responses (e.g., work of O'Connell). Other RNA modifications, such as modifications of the $5^{\prime}$ cap structure and the recently re-discovered internal $\mathrm{N}^{6}$-methyladenosine in messenger RNAs, are important in creating signatures that can distinguish between host and pathogen RNAs.

In vertebrates, and specifically in mammals, the dominant system of innate immunity is the interferon system, which was originally discovered in the 1950s as an antiviral system. RNA plays many important roles in the activation and effector arms of the interferon system. RNA mis-localization and/ or RNA elements, such as uninterrupted double stranded regions, uncapped $5^{\prime}$ termini or an incompletely modified $5^{\prime}$ caps, and modifications of the bases and/or sugars, are recognized as foreign by several receptors/RNA sensors, and this detection leads to the expression of interferon genes. Interferons, which are generally secreted from the cell that detects the foreign RNA, are soluble alarm signals that set up a latent anti-viral state in neighboring cells by inducing the expression of scores of interferon stimulated genes. Key pathways responsible for antiviral defense are latent until activated by the presence of foreign nucleic acids. For instance

(C) 2015 Garcia-Blanco This article, published in $R N A$, is available under a Creative Commons License (Attribution-NonCommercial 4.0 International), as described at http://creativecommons.org/licenses/by-nc/4.0/. 
upon binding dsRNA the family of enzymes known as $2^{\prime}-5^{\prime}$ oligoadenylate synthetases (OASs) will produce chains of adenosine residues linked via $2^{\prime}-5^{\prime}$ phosphodiester bonds (a form of ribonucleic acid) that activate a latent endonuclease, RNase L, which is required for restriction of some viruses. Interestingly, the DNA sensing cyclic GMP-AMP (cGAMP) synthase (cGAS) is homologous to OAS enzymes and leads to the synthesis of a cyclic diribonucleotide (cGAMP) that contains a $2^{\prime}-5^{\prime}$ linkage (Hornung and Hopfner). Additionally the importance of a large number of RNA binding proteins (RBPs) in setting up the interferon mediated antiviral state is becoming clear. All of this indicates a central role for RNA and RNA recognition in the interferon mediated anti-microbial response. It is interesting to note that sensing of non-self RNA (and perhaps DNA) by these systems does not involve the sequence specific reading observed with RNA interference and CRISPR/Cas immunity.

An example of the central role of RNA in the contest between genomes is the extensive use of non-coding RNAs by viral pathogens to counter immune systems of the host (see excellent reviews by J. Steitz). Pioneering work showed that the adenovirus viral associated RNA bound and inhibited the activity of the interferon inducible dsRNA-dependent protein kinase, aka PKR, and thus enhanced viral translation and propagation (Matthews, Shenk and others). A remarkable illustration of anti-innate defense RNA is the noncoding RNA produced by flaviviruses (such as those that cause dengue fever) at the expense of the majority of the progeny genomes, which are cleaved to create this potent anti-interferon RNA. Interestingly, it appears that sequences within this non-coding RNA evolve rapidly perhaps sampling many possible binding sites for host antiviral RBPs, which can then be bound and sequestered. This is consistent with evolutionary competition seen in many host-pathogen interactions.

The exploration of the role of RNA in self vs. non-self recognition is a vibrant area of investigation. The ability of RNAs to read sequences by base-pairing, to interact with proteins based on both sequence and non-sequence dependent structures or subcellular location, to serve as second messengers, to active latent enzymes, and to catalyze biochemical reactions provide this nucleic acid a plethora of ways to act in the recognition of genomes and their products. It would not be surprising to find completely new systems lurking in our cells or in the pathogens that invade them. I am optimistic that there is important new biology to be discovered in this area.

In addition to its fundamental importance, the understanding of the role of RNA in self vs. non-self recognition has vital applications to human health. The use of RNA interference to control plant pathogens can massively improve agriculture, especially in developing nations. Additionally, there are exciting programs to manipulate the RNA interference system to inhibit pathogen transmission by mosquitoes, which are second only to humans in causing disease and death around the world. The excitement in this aspect of RNA biology thus spills into applications with tangible promise for global health. 

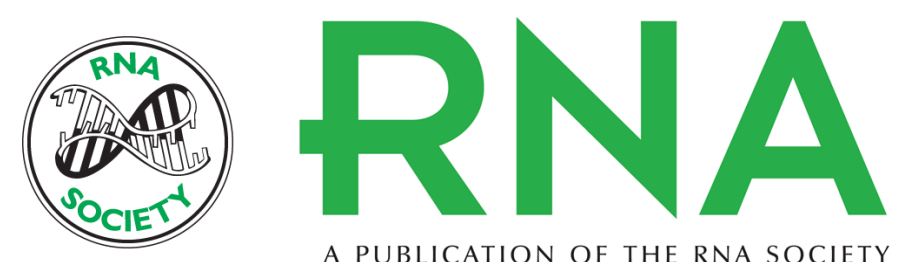

A PUBLICATION OF THE RNA SOCIETY

\section{Know thyself}

Mariano A. Garcia-Blanco

RNA 2015 21: 525-526

Open Access Freely available online through the RNA Open Access option.

Creative This article, published in RNA, is available under a Creative Commons License

Commons (Attribution-NonCommercial 4.0 International), as described at

License http://creativecommons.org/licenses/by-nc/4.0/.

Email Alerting Receive free email alerts when new articles cite this article - sign up in the box at the Service top right corner of the article or click here. 